\title{
Line Transversals of Balls and Smallest Enclosing Cylinders in Three Dimensions*
}

\author{
P. K. Agarwal, ${ }^{1}$ B. Aronov, ${ }^{2}$ and M. Sharir ${ }^{3}$ \\ ${ }^{1}$ Department of Computer Science, Box 90129, Duke University, \\ Durham, NC 27708-0129, USA \\ pankaj@cs.duke.edu \\ ${ }^{2}$ Department of Computer and Information Science, Polytechnic University, \\ Brooklyn, NY 11201, USA \\ aronov@ziggy.poly.edu \\ ${ }^{3}$ School of Mathematical Sciences, Tel Aviv University, \\ Tel Aviv 69978, Israel \\ sharir@math.tau.ac.il \\ and \\ Courant Institute of Mathematical Sciences, New York University, \\ New York, NY 10012, USA
}

\begin{abstract}
We establish a near-cubic upper bound on the complexity of the space of line transversals of a collection of $n$ balls in three dimensions, and show that the bound is almost tight, in the worst case. We apply this bound to obtain a near-cubic algorithm for computing a smallest infinite cylinder enclosing a given set of points or balls in 3-space. We also present an approximation algorithm for computing a smallest enclosing cylinder.
\end{abstract}

* All three authors have been supported by a joint grant from the U.S.-Israeli Binational Science Foundation. Pankaj Agarwal has also been supported by a National Science Foundation Grant CCR-93-01259, by an Army Research Office MURI Grant DAAH04-96-1-0013, by a Sloan Research Fellowship, and by an NYI award and matching funds from the Xerox Corporation. Boris Aronov has also been supported by NSF Grant CCR-92-11541, and by a Sloan Research Fellowship. Micha Sharir has also been supported by NSF Grants CCR-94-24398 and CCR-93-11127, by a Max-Planck Research Award, by a grant from the G.I.F., the German-Israeli Foundation for Scientific Research and Development, and by the Hermann MinkowskiMINERVA Center for Geometry at Tel Aviv University. 


\section{Introduction}

Line Transversals in Three Dimensions. Let $\mathcal{S}$ be a collection of $n$ compact convex sets with nonempty interiors in $\mathbb{R}^{3}$. A line $\ell$ is called a (line) transversal of $\mathcal{S}$ if it intersects every member of $\mathcal{S}$. Let $\mathcal{T}(\mathcal{S})$ denote the set of all line transversals of $\mathcal{S}$. Since lines in 3 -space can be parametrized by four real parameters, $\mathcal{T}(\mathcal{S})$ is a four-dimensional set. For example, one may use the parametrization $\left(\xi_{1}, \xi_{2}, \xi_{3}, \xi_{4}\right)$, where the equations of the line are given by $y=\xi_{1} x+\xi_{2}, z=\xi_{3} x+\xi_{4}$; this excludes lines parallel to the $y z$-plane, which can be treated separately, in a simpler manner, since they can be parametrized by only three real parameters. We assume that each $S \in \mathcal{S}$ has "constant description complexity," meaning that it is a semialgebraic set defined by a constant number of polynomial equalities and inequalities of constant maximum degree. In this case, $\mathcal{T}(\mathcal{S})$ is also a semialgebraic set, whose boundary $\partial \mathcal{T}(\mathcal{S})$ consists of transversals that are tangent to at least one set $S \in \mathcal{S}$. We can measure the combinatorial complexity of $\mathcal{T}(\mathcal{S})$ by the number of its faces of all dimensions, where a face is a connected component of a portion of $\partial \mathcal{T}(\mathcal{S})$ consisting of lines tangent to a fixed subset of $\mathcal{S}$. Assuming general position, a $j$-dimensional face of $\mathcal{T}(\mathcal{S})$, for $j=0,1,2,3$, consists of line transversals tangent to a fixed set of $4-j$ members of $\mathcal{S}$; see [1] and [22].

Wenger [28] obtained an $O\left(n^{4}\right)$ upper bound on the number of connected components of $\mathcal{T}(\mathcal{S})$ for the case where the sets in $\mathcal{S}$ are pairwise disjoint. Pellegrini [19]-[21] and Pellegrini and Shor [22] have studied several combinatorial and algorithmic problems involving line transversals of a set $\mathcal{S}$ of triangles and convex polytopes in 3-space. They obtained a lower bound of $\Omega\left(n^{3}\right)$ and an upper bound of $n^{3} \cdot 2^{O(\sqrt{\log n})}$ on the complexity of $\mathcal{T}(\mathcal{S})$, where $\mathcal{S}$ is a set of convex polytopes in $\mathbb{R}^{3}$ with a total of $n$ vertices. The upper bound was later improved to $O\left(n^{3} \log n\right)$ by Agarwal [1]. Pellegrini and Shor also gave an $O\left(n^{3+\varepsilon}\right)$-time algorithm, for any $\varepsilon>0$, for determining whether $\mathcal{S}$ admits any line transversal, i.e., whether $\mathcal{T}(\mathcal{S}) \neq \emptyset$. No subquartic upper bound was known for collections $\mathcal{S}$ of more general convex sets, even for collections of balls. An easier, related problem is to bound the complexity of the space of plane transversals of $\mathcal{S}$. It is shown in [3] that if each object in $\mathcal{S}$ has constant description complexity, then the combinatorial complexity of the space of plane transversals of $\mathcal{S}$ is $O\left(n^{2+\varepsilon}\right)$. See [11] and [29] for recent surveys on geometric transversal theory.

In this paper we study the case where $\mathcal{S}$ is a collection of $n$ balls in $\mathbb{R}^{3}$. We show that the complexity of $\mathcal{T}(\mathcal{S})$ is $O\left(n^{3+\varepsilon}\right)$, for any $\varepsilon>0 .{ }^{1}$ The same bound also holds if $\mathcal{S}$ if a set of $n$ homothets of a convex body of simple shape. We also show that this bound is nearly tight by exhibiting a collection $\mathcal{S}$ of $n$ balls for which the complexity of $\mathcal{T}(\mathcal{S})$ is $\Omega\left(n^{3}\right)$.

We conjecture that such a near-cubic bound on the complexity of $\mathcal{T}(\mathcal{S})$ holds for arbitrary collections $\mathcal{S}$ of $n$ simply shaped convex sets in $\mathbb{R}^{3}$. As already mentioned, this holds for the case of polyhedra.

Smallest Enclosing Cylinders. Next, we study the following problem: Let $P$ be a set of $n$ points in $\mathbb{R}^{3}$. We aim to find an infinite circular cylinder $C$ of the smallest possible radius

\footnotetext{
${ }^{1}$ Throughout this paper, $\varepsilon$ denotes an arbitrarily small positive constant. The constant of proportionality depends on $\varepsilon$, and tends to $\infty$ as $\varepsilon \downarrow 0$.
} 
that contains $P$; we refer to $C$ as a smallest enclosing cylinder of $P$. More generally, we may consider the case where $P$ is a collection of balls, and we wish to find a cylinder of smallest radius that either (a) contains all the balls in $P$, or (b) intersects every ball in $P$.

These problems arise in statistical analysis and computational metrology. In statistical analysis, given a set $P$ of points in $\mathbb{R}^{3}$, we wish to fit a line $\ell$ through $P$ so that the maximum distance between $\ell$ and the points of $P$ is minimized, which is equivalent to finding a smallest enclosing cylinder of $P$. In computational metrology, this problem arises in measuring the quality of a product [8], [10], [26], [27]. $P$ is a set of points sampled from a cylindrical object being manufactured. A smallest cylinder enclosing $P$ serves as a calibration of how close the manufactured object is to the desired shape. In order to capture measurement errors (in both statistical analysis and computational metrology), we can replace each point $p \in P$ by a ball of radius $\delta_{p}$ centered at $p$. In that case, problem (a) seeks the smallest cylinder that is guaranteed to contain all the points, regardless of their exact position; and a solution to problem (b) gives an underestimate on the radius of a smallest cylinder containing $S$.

This problem is closely related to the space of line transversals of balls in $\mathbb{R}^{3}$. Indeed, suppose, for specificity, that $P$ is the set $\left\{B\left(x_{1}, r_{1}\right), \ldots, B\left(x_{n}, r_{n}\right)\right\}$, where $B(x, r)$ denotes the closed ball of radius $r$ centered at $x$. Suppose that we conduct a binary search on the radius $r$ of the cylinder, and that we need a procedure that can determine, for a given $r$, whether there exists a cylinder of radius $r$ that contains (resp. intersects) all the balls of $S$. This decision problem is equivalent to the problem of determining whether the sets

$$
P_{r}^{-}=\left\{B\left(x_{1}, r-r_{1}\right), \ldots, B\left(x_{n}, r-r_{n}\right)\right\}
$$

and

$$
P_{r}^{+}=\left\{B\left(x_{1}, r+r_{1}\right), \ldots, B\left(x_{n}, r+r_{n}\right)\right\},
$$

respectively, have a line transversal.

This indeed is the approach that we use to solve this problem. We first present an algorithm for determining whether a given set of $n$ balls in 3-space has a line transversal. The running time of the algorithm is $O\left(n^{3+\varepsilon}\right)$, for any $\varepsilon>0$. We then combine this procedure with parametric search to obtain a near-cubic solution to computing the smallest enclosing cylinder. This improves a recent $n^{4} \log ^{O(1)} n$ solution of Schömer et al. [23].

We also present an $O\left(\left(1+1 / \delta^{2}\right) n\right)$-time algorithm to compute a cylinder enclosing a set of balls in $\mathbb{R}^{3}$, whose radius is at most $(1+\delta) r^{*}$, where $r^{*}$ is the radius of a smallest enclosing cylinder of the set. Schömer et al. [23] gave an $O\left(\left(1+1 / \delta^{2}\right) n \log 1 / \delta\right)$-time algorithm to compute a cylinder of radius $r^{*}+\delta$ enclosing a set of $n$ points in $\mathbb{R}^{3}$, assuming that the input points lie inside a unit sphere. They also presented two other algorithms for this problem that run in time $O\left(n^{4} \log (1 / \delta)\right)$ and $O\left((1+1 / \delta) n^{3} \log (1 / \delta)\right)$.

\section{Geometric Preliminaries}

In this section we introduce a few technical concepts and results that we will be using in subsequent sections. Let $\mathcal{F}$ be a set of $n d$-variate, possibly partially defined functions. The lower envelope of $\mathcal{F}$ is defined as $\mathcal{F}^{\mathrm{L}}(x)=\min _{f \in \mathcal{F}} f(x)$, and the upper envelope of $\mathcal{F}$ is defined as $\mathcal{F}^{\mathrm{U}}(x)=\max _{f \in \mathcal{F}} f(x)$; in case of partially defined functions, the 
minimum and maximum are taken only over those functions that are defined at $x$. The decomposition of $\mathbb{R}^{d}$ into maximal connected regions over each of which a fixed subset of functions attains the lower (resp. upper) envelope of $\mathcal{F}$ is called the minimization (resp. maximization) diagram of $\mathcal{F}$. A recent result of Sharir [24] shows that if the graphs of the functions in $\mathcal{F}$ have constant description complexity, i.e., if the graph of each function is a semialgebraic set defined by a constant number of polynomial equalities and inequalities of constant maximum degree, then the complexity of the lower and upper envelopes of $\mathcal{F}$ is $O\left(n^{d+\varepsilon}\right)$, for any $\varepsilon>0$. Moreover, Agarwal et al. [2] have shown that, for $d=3, \mathcal{F}$ can be preprocessed in $O\left(n^{3+\varepsilon}\right)$ time into a data structure of size $O\left(n^{3+\varepsilon}\right)$ so that, for a query point $x \in \mathbb{R}^{3}, \mathcal{F}^{\mathrm{L}}(x)$ and $\mathcal{F}^{\mathrm{U}}(x)$ and one of the functions attaining each envelope can be determined in $O\left(\log ^{2} n\right)$ time.

Let $\mathcal{B}$ be a given set of $n$ balls in $\mathbb{R}^{3}$. We use the parametrization $\left(\xi_{1}, \xi_{2}, \xi_{3}, \xi_{4}\right)$ of lines in $\mathbb{R}^{3}$ defined in the Introduction. For each ball $B \in \mathcal{B}$, let $\sigma_{B}$ denote the surface in $\mathbb{R}^{4}$ consisting of all lines tangent to $B$. We can represent $\sigma_{B}$ as the union of the graphs of two partially defined functions $\xi_{4}=F_{B}\left(\xi_{1}, \xi_{2}, \xi_{3}\right), \xi_{4}=G_{B}\left(\xi_{1}, \xi_{2}, \xi_{3}\right)$, where $F_{B}\left(\xi_{1}, \xi_{2}, \xi_{3}\right)$ (resp. $\left.G_{B}\left(\xi_{1}, \xi_{2}, \xi_{3}\right)\right)$ is the $\xi_{4}$-coordinate of the line $\left(\xi_{1}, \xi_{2}, \xi_{3}, \xi_{4}\right)$ that is tangent to $B$ from below (resp. from above). The functions $F_{B}$ and $G_{B}$ are defined whenever the line $y=\xi_{1} x+\xi_{2}$ intersects the $x y$-projection of $B$ (a condition independent of $\xi_{3}$ ); otherwise we put $F_{B}=+\infty$ and $G_{B}=-\infty$. The graphs of $F_{B}$ and $G_{B}$ are semialgebraic sets of constant description complexity. Let $\mathcal{F}=\left\{F_{B} \mid B \in \mathcal{B}\right\}$ and $\mathcal{G}=\left\{G_{B} \mid B \in \mathcal{B}\right\}$. The following observation is immediate:

Observation 2.1. A line $\left(\xi_{1}, \xi_{2}, \xi_{3}, \xi_{4}\right)$ intersects $B$ if and only if

$$
F_{B}\left(\xi_{1}, \xi_{2}, \xi_{3}\right) \leq \xi_{4} \leq G_{B}\left(\xi_{1}, \xi_{2}, \xi_{3}\right)
$$

Thus $\left(\xi_{1}, \xi_{2}, \xi_{3}, \xi_{4}\right)$ is a transversal of $\mathcal{B}$ if and only if

$$
\max _{B \in \mathcal{B}} F_{B}\left(\xi_{1}, \xi_{2}, \xi_{3}\right) \leq \xi_{4} \leq \min _{B \in \mathcal{B}} G_{B}\left(\xi_{1}, \xi_{2}, \xi_{3}\right) .
$$

In other words, $\mathcal{T}(\mathcal{B})$ is the region "sandwiched" between the upper envelope $\mathcal{F}^{\mathrm{U}}$ of $\mathcal{F}$ and the lower envelope $\mathcal{G}^{\mathrm{L}}$ of $\mathcal{G}$.

Since the functions in $\mathcal{F}, \mathcal{G}$ are partial trivariate algebraic functions, of constant description complexity, the result of Agarwal et al. [2] mentioned at the beginning of this section implies the following fact.

Observation 2.2. A set $\mathcal{B}$ of $n$ balls in $\mathbb{R}^{3}$ can be preprocessed in time $O\left(n^{3+\varepsilon}\right)$, for any $\varepsilon>0$, into a data structure of size $O\left(n^{3+\varepsilon}\right)$, so that we can determine in $O\left(\log ^{2} n\right)$ time whether a query line $\ell$ is a transversal of $\mathcal{B}$.

This observation, however, falls short of giving a bound on the complexity of $\mathcal{T}(\mathcal{B})$. In order to bound this complexity, we need a bound on the complexity of the "sandwich" region enclosed between the lower envelope $\mathcal{G}^{\mathrm{L}}$ and the upper envelope $\mathcal{F}^{\mathrm{U}}$. It is still an open problem whether a near-cubic bound holds for the case of trivariate functions. However, such a result was recently established in [3] for bivariate functions, and is used in the next section: 
Theorem 2.3 [3]. The complexity of the region enclosed between the upper envelope of $n$ bivariate functions of constant description complexity and the lower envelope of $n$ other bivariate functions of constant description complexity is $O\left(n^{2+\varepsilon}\right)$, for any $\varepsilon>0$. This region can be computed, in an appropriate model of computation, in time $O\left(n^{2+\varepsilon}\right)$, for any $\varepsilon>0$.

\section{Complexity of the Set of Line Transversals}

Let $\mathcal{B}$ be a set of $n$ balls of arbitrary radii in $\mathbb{R}^{3}$. In this section we bound the combinatorial complexity of $\mathcal{T}(\mathcal{B})$. The following theorem states the main result.

Theorem 3.1. Let $\mathcal{B}$ be a collection of $n$ balls in $\mathbb{R}^{3}$. Then the complexity of $\mathcal{T}(\mathcal{B})$ is $O\left(n^{3+\varepsilon}\right)$, for any $\varepsilon>0$.

Proof. We assume that the balls in $\mathcal{B}$ are in general position, i.e., that no line is tangent to more than four balls, and that the point $\left(\xi_{1}, \xi_{2}, \xi_{3}, \xi_{4}\right)$ corresponding to a line tangent to exactly $k$ balls, $k=1, \ldots, 4$, lies in a face of codimension $k$ in the arrangement of the set of surfaces $\left\{\sigma_{B} \mid B \in \mathcal{B}\right\}$. Following the same argument as in [24], the upper bound can be extended to the situation when the balls are not in general position.

For a set $\mathcal{B}$ of balls in general position, a simple counting argument shows that the number of faces of $\mathcal{T}(\mathcal{B})$ that are not incident to any vertex of $\mathcal{T}(\mathcal{B})$ is bounded by $O\left(n^{3}\right)$, and that the number of all other faces of $\mathcal{T}(\mathcal{B})$ is proportional to the number of vertices in $\mathcal{T}(\mathcal{B})$. It thus suffices to bound the number of vertices in $\mathcal{T}(B)$. We refer to a transversal as extreme if it is tangent to four balls in $\mathcal{B}$, so that it corresponds to a vertex of $\mathcal{T}(\mathcal{B})$ (where four of the graphs of the functions $F_{B}, G_{B}$ intersect).

Let the set $\mathcal{B}=\left\{B_{1}, \ldots, B_{n}\right\}$ of balls be ordered so that the radii $r_{i}$ of the balls $B_{i}$ form a nonincreasing sequence. Fix a direction $\mathbf{n}$ in $\mathbb{R}^{3}$ not parallel to the $x$-axis, and consider the family of all lines in direction $\mathbf{n}$. Let $H_{\mathbf{n}}$ be some plane orthogonal to $\mathbf{n}$, and let $B^{(\mathbf{n})}$ denote the orthogonal projection of a ball $B$ to $H_{\mathbf{n}}$. Let $\mathcal{B}^{(\mathbf{n})}$ denote the collection of $n$ disks in $H_{\mathbf{n}}$ resulting from the projection of the balls in $\mathcal{B}$.

Suppose that $\ell$ is an extreme transversal of $\mathcal{B}$ in direction $\mathbf{n}$, and let $B_{i_{1}}, B_{i_{2}}, B_{i_{3}}, B_{i_{4}}$ be the four balls of $\mathcal{B}$ to which $\ell$ is tangent. Without loss of generality, assume that $i_{1}<i_{2}<i_{3}<i_{4}$, i.e., $B_{i_{1}}$ has the largest radius among these four balls. Clearly, the point $\ell \cap H_{\mathbf{n}}$ must lie in each of the disks of $\mathcal{B}^{(\mathbf{n})}$ and on the boundary of the four disks $B_{i_{k}}^{(\mathbf{n})}$, for $k=1, \ldots, 4$. Our strategy is to fix the ball $B_{i_{1}}$, and to analyze the number of extreme transversals $\ell$ with these properties, that is,

$(\star)$ extreme transversals that are tangent to $B_{i_{1}}$ and to three other balls of $\mathcal{B}$ whose indices are larger than $i_{1}$.

We will show that the number of such extreme transversals is $O\left(n^{2+\varepsilon}\right)$, for any $\varepsilon>0$. This will clearly imply the bound asserted in the theorem. In fact, we will count the number of extreme transversals of $\mathcal{B}_{i_{1}}=\left\{B_{i_{1}}, B_{i_{1}+1}, \ldots, B_{n}\right\}$ that are tangent to $B_{i_{1}}$. Since any transversal of $\mathcal{B}$ satisfying property $(\star)$ is also an extreme transversal of $\mathcal{B}_{i_{1}}$, what we are counting is a superset of transversals satisfying property $(\star$ ) (clearly, not every extreme transversal of $\mathcal{B}_{i_{1}}$ is necessarily a transversal of $\mathcal{B}$ satisfying $(\star)$ ). 


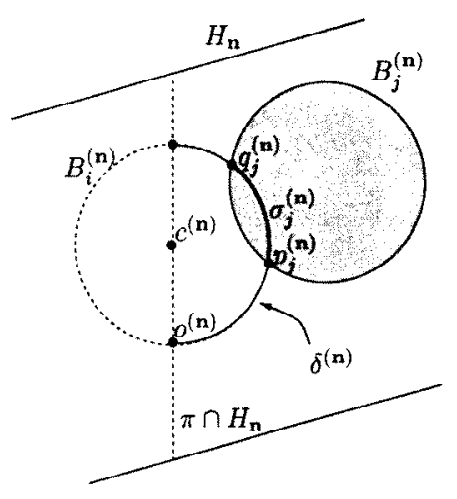

Fig. 1. Projection of $B_{i}$ and $B_{j}$ onto $H_{\mathbf{n}}$.

Fix a ball $B_{i}$, for some $i=1, \ldots, n-3$, and let $\mathbf{n}$ be an arbitrary direction not parallel to the $x$-axis. Intersect $B_{i}^{(\mathbf{n})}$ with the plane $\Pi$ passing through its center and parallel to the $y z$-plane (since we have excluded the case in which $\mathbf{n}$ is parallel to the $x$-axis, $\left.B_{i}^{(\mathbf{n})} \not \subset \Pi\right)$. Let $\delta^{(\mathbf{n})}$ denote the semicircle bounding the portion of $B_{i}^{(\mathbf{n})}$ that lies in the positive half-space bounded by $\Pi$. Let $c^{(\mathbf{n})}, o^{(\mathbf{n})}$ denote the center of $B_{i}^{(\mathbf{n})}$ and the clockwise endpoint of $\delta^{(\mathbf{n})}$ (when viewed in direction $\mathbf{n}$ ), respectively. For a point $p \in \delta^{(\mathbf{n})}$, we define $\varphi^{(\mathbf{n})}(p)=\tan \left(\left(\angle o^{(\mathbf{n})} c^{(\mathbf{n})} p\right) / 2\right)$. Since $0 \leq \angle o^{(\mathbf{n})} c^{(\mathbf{n})} p \leq \pi, \varphi^{(\mathbf{n})}$ increases monotonically with the length of the $\operatorname{arc}$ of $\delta^{(\mathbf{n})}$ from $o^{(\mathbf{n})}$ to $p$. See Fig. 1.

For each $j>i$, we define two functions $f_{j}(\mathbf{n}), g_{j}(\mathbf{n})$, as follows. Let $\sigma_{j}^{(\mathbf{n})}=\delta^{(\mathbf{n})} \cap B_{j}^{(\mathbf{n})}$. Since $r_{j} \leq r_{i}, \sigma_{j}^{(\mathbf{n})}$ must be a (possibly empty) connected arc. Let $p_{j}^{(\mathbf{n})}, q_{j}^{(\mathbf{n})}$ denote the clockwise and counterclockwise endpoints of $\sigma_{j}^{(\mathbf{n})}$, respectively. We define $f_{j}(\mathbf{n})=$ $\varphi^{(\mathbf{n})}\left(p_{j}^{(\mathbf{n})}\right)$ and $g_{j}(\mathbf{n})=\varphi^{(\mathbf{n})}\left(q_{j}^{(\mathbf{n})}\right)$. If $\sigma_{j}^{(\mathbf{n})}$ is empty, we put $f_{j}(\mathbf{n})=\infty$ and $g_{j}(\mathbf{n})=0$. It is a routine task to show that the graphs of $f_{j}$ and $g_{j}$ are semialgebraic sets of constant description complexity, provided that $\mathbf{n}$ is specified appropriately.

Let $\ell$ be an extreme transversal in direction $\mathbf{n}$ that is tangent to $B_{i}$ and to three other balls with larger indices, such that $\ell \cap H_{\mathbf{n}}$ lies in $\delta^{(\mathbf{n})}$. Then the parameter $t=\varphi^{(\mathbf{n})}\left(\ell \cap H_{\mathbf{n}}\right)$ must satisfy

$$
\max _{j>i} f_{j}(\mathbf{n}) \leq t \leq \min _{j>i} g_{j}(\mathbf{n}) .
$$

Moreover, the point (n, $t$ ) must be a vertex of the region enclosed between the upper envelope of the $f_{j}$ 's and the lower envelope of the $g_{j}$ 's, since it lies on (at least) three of the surfaces and the balls in $\mathcal{B}$ are assumed to be in general position. By Theorem 2.3, the number of such vertices is $O\left(n^{2+\varepsilon}\right)$, for any $\varepsilon>0$.

Repeating the same analysis, but replacing $\delta^{(\mathbf{n})}$ by the other semicircle bounding $B_{i}^{(\mathbf{n})}$, we conclude that the number of extreme transversals that are tangent to $B_{i}$ and to three other balls with larger indices is $O\left(n^{2+\varepsilon}\right)$, for any $\varepsilon>0$. As observed above, this completes the proof of the theorem.

Remark 3.2. The crucial observation in the above proof is that, for every direction $\mathbf{n}$, 
$\sigma_{j}^{(\mathbf{n})}=\delta^{(\mathbf{n})} \cap B_{j}^{(\mathbf{n})}$ is a connected arc. A similar property also holds for a set of homothets of a compact convex set. More precisely, let $\mathcal{S}$ be a set of $n$ homothets of a semialgebraic compact convex set of constant description complexity in $\mathbb{R}^{3}$. Assume that the sets in $\mathcal{S}$ are sorted in a nonincreasing order of their scaling factors. For a direction $\mathbf{n}$, let $S_{i}^{(\mathbf{n})}$ denote the projection of $S_{i}$ onto a plane $H_{\mathbf{n}}$ orthogonal to $\mathbf{n}$, and let $\Pi_{\mathbf{n}}$ be the plane parallel to the $y z$-plane for which the length of $\Pi_{\mathbf{n}} \cap S_{i}^{(\mathbf{n})}$ is maximized over all planes parallel to the $y z$-plane. Let $\delta^{(\mathbf{n})}$ be the portion of $\partial S_{i}^{(\mathbf{n})}$ lying in the closed positive halfspace bounded by $\Pi_{\mathbf{n}}$. Then, for any $j>i, \delta^{(\mathbf{n})} \cap S_{j}^{(\mathbf{n})}$ is a connected arc. This follows from the observations that (a) $\partial S_{i}^{(\mathbf{n})}$ and $\partial S_{j}^{(\mathbf{n})}$, being homothetic to each other, intersect at two points [13], and (b) if these two points lie on $\delta^{(\mathbf{n})}$, then, $S_{j}^{(\mathbf{n})} \cap \partial S_{i}^{(\mathbf{n})} \subseteq \delta^{(\mathbf{n})}$, for otherwise $S_{j}^{(\mathbf{n})} \cap \Pi_{\mathbf{n}}$ would be longer than $S_{i}^{(\mathbf{n})} \cap \Pi_{\mathbf{n}}$, which is impossible since $S_{j}^{(\mathbf{n})}$ has a smaller scaling factor than that of $S_{i}^{(\mathbf{n})}$. Now an easy modification of the above proof shows that the complexity of $\mathcal{T}(\mathcal{S})$ is also $O\left(n^{3+\varepsilon}\right)$, for any $\varepsilon>0$.

\section{Testing for the Existence of a Line Transversal}

Let $\mathcal{B}=\left\{B_{1}, \ldots, B_{n}\right\}$ be a collection of $n$ balls in 3 -space in general position, as described in the beginning of the proof of Theorem 3.1. We present a near-cubic-time algorithm for determining whether $\mathcal{T}(\mathcal{B}) \neq \emptyset$. If $n<4$, we can explicitly compute $\mathcal{T}(\mathcal{B})$ in $O$ (1) time, so assume $n \geq 4$.

We begin by extending the notion of extreme transversals so that there always exists an extreme transversal whenever $\mathcal{T}(\mathcal{B}) \neq \emptyset$.

Recall the parametrization $\left(\xi_{1}, \xi_{2}, \xi_{3}, \xi_{4}\right)$ of the set of lines, as defined in the Introduction. Then $\mathcal{T}(\mathcal{B})$ is the region "sandwiched" between two envelopes in $\mathbb{R}^{4}$. Consider a connected component $C$ of the sandwich region and let $w$ be the highest point of $C$, if such a point exists (the remaining case will be discussed below). Then $w$ is a vertex, a $\xi_{4}$ extreme point on a curve, a $\xi_{4}$-extreme point on a two-dimensional face, or a $\xi_{4}$-extreme point on a three-dimensional face. We extend the definition of extreme transversals, so that they also include lines that correspond to these points. For example, the second type corresponds to a line transversal of $\mathcal{B}$ touching three fixed balls and, among nearby such transversals, locally maximizing $\xi_{4}$. Clearly the number of extreme transversals that are not vertices of $\mathcal{T}(\mathcal{B})$ (i.e., the extreme transversals that are tangent to fewer than four balls) is $O\left(n^{3}\right)$ and a superset of them can be trivially computed in $O\left(n^{3}\right)$ time, by considering all individual surfaces, intersections of pairs and intersections of triples of surfaces, and by computing locally $\xi_{4}$-extreme points on each such set (assuming general position, there are only $O(1)$ such points on each of these sets).

Suppose $C$ is unbounded in the $\xi_{4}$-direction. Then $C$ contains a transversal parallel to the $y z$-plane: The unboundedness implies that there are lines in $C$ whose projection on the $x z$-plane has arbitrarily large $z$-intercept. The projection of such a transversal must be nearly vertical (in the $x z$-plane), because of the compactness of the balls. As $\mathcal{T}(\mathcal{B})$ is a closed set, in the limit we get a line transversal orthogonal to the $x$-axis.

In the three-dimensional space of lines perpendicular to the $x$-axis, we choose another parametrization and repeat the process, identifying local extrema in the last parameter. It is easily verified that no more than $O\left(n^{3}\right)$ potential extreme transversals can be generated 
by carrying out this process to its logical conclusion; a superset of these transversals, of size $O\left(n^{3}\right)$, can be computed in $O\left(n^{3}\right)$ time.

The following lemma follows from the definition of extreme transversals given above.

Lemma 4.1. If $\mathcal{T}(\mathcal{B})$ is nonempty, then each connected component of $\mathcal{T}(\mathcal{B})$ contains at least one extreme transversal of $\mathcal{B}$, in the extended sense just defined. Moreover, if $\mathcal{T}(\mathcal{B})$ has nonempty interior, then the closure of each connected component of its interior contains at least one point corresponding to an extreme transversal.

In view of the lemma, the emptiness of $\mathcal{T}(\mathcal{B})$ can be tested by determining whether there exists an extreme transversal of $\mathcal{B}$. The algorithm works in two phases. In the first phase we compute a superset of extreme transversals (i.e., we compute a set of lines that are guaranteed to contain all extreme transversals of $\mathcal{B}$ ), and in the second phase we check whether any of them is indeed a transversal of $\mathcal{B}$. As discussed above, there are $O\left(n^{3}\right)$ lines that touch fewer than four balls and could potentially be extreme transversals of $\mathcal{B}$; they can be computed in $O\left(n^{3}\right)$ time. Let $\mathcal{L}_{1}$ denote the set of these lines. (This set also includes extreme lines perpendicular to the $x$-axis.)

We next compute a set $\mathcal{L}_{2}$ of $O\left(n^{3+\varepsilon}\right)$ lines that touch four balls of $\mathcal{B}$ and include all extreme transversals that correspond to vertices of $\mathcal{T}(\mathcal{B})$, by proceeding along the same lines as the proof of Theorem 3.1. For each $B_{i}$ (and for each of the two semicircles, $\delta^{(\mathbf{n})}$ and its complement, in the corresponding planes of projection), we define the two collections of bivariate functions, $\left\{f_{j}(\mathbf{n})\right\}_{j>i}$, and $\left\{g_{j}(\mathbf{n})\right\}_{j>i}$, as in the preceding section. Let $\ell$ be an an extreme transversal that is tangent to $B_{i}$ at a point that projects to the semicircle under consideration and that is also tangent to three other balls with larger indices. Then $\ell$ corresponds to a vertex of the region sandwiched between the lower envelope of the $g_{j}$ 's and the upper envelope of the $f_{j}$ 's. Using the algorithm described in [3] (see Theorem 2.3), we can compute all these vertices in time $O\left(n^{2+\varepsilon}\right)$, for any $\varepsilon>0$. We repeat this step for each ball $B_{i}$ and for each of the two corresponding semicircles, and obtain, in overall time $O\left(n^{3+\varepsilon}\right)$, for any $\varepsilon>0$, a set of lines that contains all extreme line transversals corresponding to vertices of $\mathcal{T}(\mathcal{B})$.

Let $\mathcal{L}=\mathcal{L}_{1} \cup \mathcal{L}_{2}$ be the set of all lines computed by the above procedures. As already observed, some of the lines may not be transversals to the entire set of balls, so we need to test whether any of these lines is indeed a transversal of $\mathcal{B}$. By Observation 2.2, we can preprocess $\mathcal{B}$, in $O\left(n^{3+\varepsilon}\right)$ time, into a data structure of size $O\left(n^{3+\varepsilon}\right)$, so that we can determine in $O\left(\log ^{2} n\right)$ time whether a line $\ell \in \mathcal{L}$ is a transversal of $\mathcal{B}$. Hence, we can identify all extreme transversals of $\mathcal{B}$ in $O\left(n^{3+\varepsilon}\right)$ additional time, for any $\varepsilon>0$. We therefore conclude:

Theorem 4.2. Given a set $\mathcal{B}$ of $n$ balls in 3-space in general position, we can determine in $O\left(n^{3+\varepsilon}\right)$ time, for any $\varepsilon>0$, whether $\mathcal{B}$ admits a line transversal.

Remark 4.3. (i) If all balls in $\mathcal{B}$ have the same radius, then, for each $i$, we can compute the region lying between the lower envelope of $\left\{g_{j}(\mathbf{n}) \mid 1 \leq j \neq i \leq n\right\}$ and the upper envelope of $\left\{f_{j}(\mathbf{n}) \mid 1 \leq j \neq i \leq n\right\}$. In this case the collection of these sandwich regions represents the boundary of $\mathcal{T}(\mathcal{B})$. It is therefore sufficient to determine whether 
any of these sandwich regions is nonempty (and thus the second "filtering" phase is not required).

(ii) The above algorithm can handle some limited degeneracies in the input. For example, the algorithm works even if more than four balls are tangent to a line, as long as the number of such balls is bounded by a constant. We need this extension in 6 .

\section{Lower Bound}

In this section we describe a construction of a family $\mathcal{B}$ of $n$ balls in $\mathbb{R}^{3}$, for which $\mathcal{T}(\mathcal{B})$ has complexity $\Omega\left(n^{3}\right)$. We also present an easier construction of a set $\mathcal{B}$ of $n$ balls of the same radius, with $\mathcal{T}(\mathcal{B})$ consisting of $\Omega\left(n^{2}\right)$ connected components. The constructions are inspired by those of Pellegrini [21].

We begin by describing the second, simpler construction. Consider a plane $\pi$ through the origin $O$ in $\mathbb{R}^{3}$. Let $R \gg 1$ be a quantity to be fixed later. Place two balls of radius $R$ tangent to $\pi$ at $O$ from the opposite sides of $\pi$, and then move them apart, perpendicular to $\pi$, so that they lie at distance 1 from $\pi$. Denote the resulting two balls by $B_{\pi}^{1}$ and $B_{\pi}^{2}$. Note that no line transversal of $\left\{B_{\pi}^{1}, B_{\pi}^{2}\right\}$ is parallel to $\pi$. Moreover, any line that passes through $O$ and makes an angle larger than $\varepsilon=\varepsilon(R)$ with $\pi$ is a transversal of this set. Note that $\varepsilon(R) \rightarrow 0$ as $R \rightarrow \infty$.

Consider a set of $n / 2$ planes $\left\{\pi_{1}, \ldots, \pi_{n / 2}\right\}$ passing through the origin, with no three planes sharing a common line. Put

$$
\mathcal{B}=\left\{B_{\pi_{i}}^{1}, B_{\pi_{i}}^{2} \mid i=1, \ldots, n / 2\right\} .
$$

We claim that $\mathcal{T}(\mathcal{B})$ has $\Omega\left(n^{2}\right)$ connected components, for some sufficiently large $R$. Indeed, no line parallel to any of the planes is a transversal of $\mathcal{B}$. Let $\mathbb{S}$ denote the sphere of directions in $\mathbb{R}^{3}$ - the unit-radius sphere centered at the origin; each direction in 3-space can be represented as a point on $\mathbb{S}$. The locus of orientations of lines parallel to $\pi_{i}$ is a great circle $C_{i}=C\left(\pi_{i}\right)$ on $\mathbb{S}$, and the $n / 2$ great circles $\left\{C_{1}, \ldots, C_{n / 2}\right\}$ in general position corresponding to the $n / 2$ planes $\left\{\pi_{1}, \ldots, \pi_{n / 2}\right\}$ induce an arrangement $\mathcal{A}$ on $\mathbb{S}$ with $\Theta\left(n^{2}\right)$ faces. To finish the argument it is sufficient to show that, for a sufficiently large $R$, each face of $\mathcal{A}$ contains at least one line orientation corresponding to a transversal of $\mathcal{B}$. Indeed, any line passing through $O$ and making an angle more than $\varepsilon$ with the planes is a transversal of $\mathcal{B}$. Therefore every point on $\mathbb{S}$ outside the union of $n / 2$ bands of half-width $\varepsilon$, each centered around one of the great circles $C_{i}$, is the orientation of a line transversal to $\mathcal{B}$ through $O$. Picking $R$ large enough we can assure that the bands are sufficiently narrow so that every face of $\mathcal{A}$ contains a point outside of their union. This completes the construction of a family of balls for which $\mathcal{T}(\mathcal{B})$ has $\Omega\left(n^{2}\right)$ connected components.

To obtain $\mathcal{T}(\mathcal{B})$ with a large number of extreme transversals, we proceed as follows: For any $\delta>0$, let $K_{\delta} \subseteq \mathbb{S}$ be the closed spherical cap centered at the north pole $(0,0,1)$ with angular opening $2 \delta$, i.e., $K_{\delta}$ is the set of unit vectors whose angles with the positive $z$-axis are at most $\delta$. Let $\left\{\pi_{1}, \ldots, \pi_{n / 4}\right\}$ be a set of $n / 4$ planes passing through the origin and let $C=\left\{C\left(\pi_{i}\right) \mid i=1, \ldots, n / 4\right\}$ be the set of great circles as defined in the previous paragraph. We choose the planes so that most (i.e., $\Theta\left(n^{2}\right)$ ) of the faces of the arrangement of great circles $\mathcal{A}=\mathcal{A}(C)$ on $\mathbb{S}$ lie in a spherical cap $K_{\delta}$ centered at the north pole, 
for some $\delta \ll 1$. Clearly this requires a choice of $\varepsilon<\delta / n$ and a correspondingly large $R$. (Notice also that $\mathcal{A}$ is centrally symmetric, with center at the origin. Two opposite points of $\mathbb{S}$ correspond to lines with opposite orientation. So, in fact, we are considering an arrangement in which two opposite spherical caps contain most of the features.) Let $\mathcal{B}^{\prime}=\left\{B_{\pi_{i}}^{1}, B_{\pi_{i}}^{2} \mid i=1, \ldots, n / 4\right\}$ be the resulting set of $n / 2$ spheres.

Consider now the set of lines passing through a point $O^{\prime}$ near $O$. The set of orientations of lines missing both balls $B_{\pi_{i}}^{1}, B_{\pi_{i}}^{2}$ and passing through $O^{\prime}$ is a slightly distorted version of the band of half-width $\varepsilon$ around the great circle $C_{i}$. Indeed, the orientations of the lines tangent to $B_{\pi_{i}}^{1}$ and passing through $O^{\prime}$ sweep out a cone corresponding to a circle near the great circle $C_{i}$, so the claim follows. In particular, the orientations of all transversals of $\mathcal{B}$ passing through $O^{\prime}$ is a complement of a set of narrow (in general, asymmetric) bands around the same family of great circles $\left\{C_{i}\right\}$. It is easy to verify that there are a sufficiently small neighborhood $N$ of $O$ and a choice of $R$, so that the following holds: There is a fixed set of $\Theta\left(n^{2}\right)$ orientations $\theta$, one in each face of $\mathcal{A}$ contained in the spherical cap $K_{\delta}$, so that each $\theta$ corresponds to a different component of $\mathcal{T}\left(\mathcal{B}^{\prime}\right)$ and such that for every point $O^{\prime} \in N$ the line passing through $O^{\prime}$ in direction $\theta$ is a transversal of $\mathcal{B}^{\prime}$.

Now we construct a collection $\mathcal{B}^{\prime \prime}=\left\{B_{1}^{\prime \prime}, \ldots, B_{n / 2}^{\prime \prime}\right\}$ of $n / 2$ additional small congruent balls around $O$, so that the following conditions hold:

1. For each $i=1, \ldots, n / 2, B_{i}^{\prime \prime}$ is contained in $N$ and the distance from $O$ to the center of $B_{i}^{\prime \prime}$ is half the common radius of these balls.

2. The centers of the balls lie at the vertices of a regular $(n / 2)$-gon in the $x y$-plane centered at $O$.

Put $I=\bigcap_{i} B_{i}^{\prime \prime}$ and $\mathcal{B}=\mathcal{B}^{\prime} \cup \mathcal{B}^{\prime \prime}$. Note that $I$ is a scaled copy of a set $I_{0}$ that depends only on $n$ and not on $\delta$. Fix a face $f \subset K_{\delta}$ of $\mathcal{A}$. The preceding discussion implies that there is a connected component $Q$ of $\mathcal{T}(\mathcal{B})$ that contains only lines whose orientation lies in $f$. We claim that each such component $Q$ contains at least $n / 2$ distinct two-dimensional faces of $\mathcal{T}(\mathcal{B})$ on its boundary. This implies the claimed lower bound, since the number of faces $f$ in $K_{\delta}$ is $\Theta\left(n^{2}\right)$.

As observed above, there exists an orientation $\theta_{f} \in f$ so that for all points $O^{\prime} \in I$ the line $\ell\left(O^{\prime}, \theta_{f}\right)$ through $O^{\prime}$ in direction $\theta_{f}$ is a transversal of $\mathcal{B}^{\prime}$ and thus of $\mathcal{B}$. Let $I^{*}$ denote the projection of $I$ onto a plane perpendicular to $\theta$. $I^{*}$ is a scaled copy of the corresponding projection of $I_{0}$. Since $\theta$ is near-vertical, $I^{*}$ has the shape of a convex " $n / 2$-gon" bounded by circular arcs, provided $\delta$ is sufficiently small. For any vertex $v_{i}$, for $i=1, \ldots, n / 2$, of this "circular polygon," $\ell\left(v_{i}, \theta_{f}\right)$ is a transversal of $\mathcal{B}$ lying on the boundary of $\mathcal{T}(\mathcal{B})$. For every $i, \ell\left(v_{i}, \theta_{f}\right)$ touches a different pair of balls of $\mathcal{B}^{\prime \prime}$, and does not touch any other ball of $\mathcal{B}^{\prime \prime}$. Therefore, the lines $\left\{\ell\left(v_{i}, \theta_{f}\right) \mid i=1, \ldots, n / 2\right\}$ are witnesses of the presence of $n / 2$ different two-dimensional faces on the boundary of $Q$, which completes the argument. Hence we have shown:

\section{Theorem 5.1.}

(i) There exists a set of $n$ balls in $\mathbb{R}^{3}$ whose transversal space has $\Omega\left(n^{3}\right)$ complexity.

(ii) There exists a set of $n$ unit-radius balls in $\mathbb{R}^{3}$, for which the space of line transversals consists of $\Omega\left(n^{2}\right)$ connected components. 


\section{Computing a Smallest Enclosing Cylinder}

Let $\mathcal{B}=\left\{B\left(x_{1}, r_{1}\right), \ldots, B\left(x_{n}, r_{n}\right)\right\}$ be a set of $n$ balls in 3-space, where $B\left(x_{i}, r_{i}\right)$ is the ball of radius $r_{i}$ centered at $x_{i}$ and $r_{i} \geq r_{j}$ whenever $i \leq j$. We assume that the balls in $\mathcal{B}$ are in general position in the sense that any cylinder is tangent to at most five balls of $\mathcal{B}$. We wish to compute a smallest cylinder containing all balls of $\mathcal{B}$. (The case of a smallest cylinder that intersects all the balls in $\mathcal{B}$ can be handled in essentially the same manner.) For a real parameter $r \geq \max _{i} r_{i}$, let $\mathcal{B}_{r}^{-}=\left\{B\left(x_{i}, r-r_{i}\right) \mid 1 \leq i \leq n\right\}$, and recall that there exists a cylinder of radius $r$ containing all balls of $\mathcal{B}$ if and only if $\mathcal{B}_{r}^{-}$has a line transversal. By our assumption, a transversal of $\mathcal{B}_{r}^{-}$is tangent to at most five balls. We thus need to find the smallest value $r^{*}$ of $r$ such that $\mathcal{B}_{r}^{-}$admits a line transversal. We accomplish this by applying the parametric search technique of Megiddo [15]. First, we need an "oracle" for determining whether a given $r$ is equal to, greater than, or smaller than $r^{*}$. This can be done using Theorem 4.2. In fact, Theorem 4.2 can be extended so that it can also determine whether a given $r$ is equal to $r^{*}$, as follows. It can be shown that the interior of $\mathcal{T}\left(\mathcal{B}_{r}^{-}\right)$is empty if and only if $r=r^{*}$. In view of Lemma 4.1, it suffices to test for the emptiness condition locally near each extreme transversal $\ell$. This local test has to consider only the $O(1)$ balls to which $\ell$ is tangent (by our general-position assumption, at most five balls are tangent to $\ell$ ), and to determine whether the interior of the space of line transversals of these balls is empty; this can be done in constant time. The oracle thus runs in $O\left(n^{3+\varepsilon}\right)$ time, for any $\varepsilon>0$.

Next, we simulate the first phase of the oracle (i.e., the algorithm that computes a superset of extreme transversals) generically on $\mathcal{B}_{r^{*}}^{-}$, without knowing the value of $r^{*}$, and maintain an interval $I$ that is guaranteed to contain $r^{*}$; initially, $I$ is set to $\left[\max _{i} r_{i}, \infty\right.$ ]. The algorithm for computing extreme transversals, as described above, is comparisonbased, in the sense that its control flow is governed by comparisons, each of which amounts to testing the sign of some low-degree polynomial in $r^{*}$. Moreover, the algorithm uses the value of $r^{*}$ only to resolve such comparisons. Since $r^{*}$ is unknown, resolving a comparison involves determining the sign of a corresponding polynomial $p(r)$ at $r=r^{*}$. Whenever the simulation of the algorithm encounters such a comparison, we compute the roots $\rho_{1}, \ldots, \rho_{k}$ of the corresponding polynomial $p(r)$. Applying Theorem 4.2 to $\mathcal{B}_{r_{i}}^{-}$, for each $1 \leq i \leq k$, we determine, in $O\left(n^{3+\varepsilon}\right)$ time, whether $\rho_{i}=r^{*}, \rho_{i}<r^{*}$, or $\rho_{i}>r^{*}$. If any $\rho_{i}$ is equal to $r^{*}$, we stop the entire procedure. Otherwise, we have an interval $\left(\rho_{i}, \rho_{i+1}\right)$ that contains $r^{*}$. We compute the sign of $p\left(r^{*}\right)$, by evaluating $p(r)$ at any point in $\left(\rho_{i}, \rho_{i+1}\right)$, and thus resolve the comparison. Next, we shrink the interval $I$ to $I \cap\left(\rho_{i}, \rho_{i+1}\right)$, and continue the simulation of the generic algorithm.

The traditional parametric search should simulate both phases of the algorithm, but the following lemma shows that simulating the first phase is sufficient, in the sense that the algorithm always terminates before finishing the simulation of the first phase. (Recall that if all balls in $\mathcal{B}$ have the same radius, then the second phase is not required anyway.)

Lemma 6.1. The generic algorithm that simulates the first phase of the oracle always performs a comparison whose corresponding polynomial vanishes at $r^{*}$.

Proof. Suppose that the generic algorithm does not make such a comparison. Then, 
at the end of the simulation we have an open interval $I_{f}$ containing $r^{*}$. Since $r$ is an indeterminate in the simulation, each potential extreme transversal computed by the generic algorithm can be parametrized as $\ell(r)=\left(\xi_{1}(r), \xi_{2}(r), \xi_{3}(r), \xi_{4}(r)\right)$ for $r \in I_{f}$, where the $\xi_{i}$ 's are univariate algebraic functions of $r$. Each such line $\ell(r)$ is tangent to the same subset of balls $B\left(x_{i_{1}}, r-r_{i_{1}}\right), \ldots, B\left(x_{i_{k}}, r-r_{i_{k}}\right)$, for every $r \in I_{f}$. Let $\mathcal{L}$ be the set of parametrized lines computed by the generic algorithm. Then, for any $r_{0} \in I_{f}$, the potential extreme transversals of $\mathcal{B}_{r_{0}}^{-}$computed by (the nongeneric version of) the first phase is the set $\mathcal{L}\left(r_{0}\right)=\left\{\ell\left(r_{0}\right) \mid \ell \in \mathcal{L}\right\}$.

Let $\ell_{0}$ be an extreme transversal line of $\mathcal{B}_{r^{*}}^{-}$, and let $\ell_{0}(r)$ be the corresponding parametrized line in $\mathcal{L}$ (so that $\ell_{0}=\ell_{0}\left(r^{*}\right)$ ). For the sake of simplicity, assume that $\ell_{0}$ is a vertex of $\mathcal{T}\left(\mathcal{B}_{r^{*}}^{-}\right)$, which is an intersection point of four surfaces. The generic algorithm computes a superset of extreme transversals of $\mathcal{B}_{r^{*}}^{-}$, so $\ell_{0} \in \mathcal{L}$. Since $r^{*}$ is the radius of a smallest enclosing cylinder of $\mathcal{B}, \ell_{0}(r)$ is not a transversal of $\mathcal{B}_{r}^{-}$, for any $r<r^{*}$. Let $B\left(x_{i}, r^{*}-r_{i}\right)$ be the largest (i.e., the first in the list) ball that $\ell_{0}$ is tangent to, so $\ell_{0}$ intersects the interiors of $B\left(x_{1}, r^{*}-r_{1}\right), \ldots, B\left(x_{i-1}, r^{*}-r_{i-1}\right)$. Hence, for any $r_{0} \in I_{f}$ smaller than $r^{*}, \ell_{0}\left(r_{0}\right)$ still intersects the interiors of $B\left(x_{1}, r_{0}-r_{1}\right), \ldots, B\left(x_{i-1}, r_{0}-r_{i-1}\right)$. However, by definition of $r^{*}, \ell_{0}\left(r_{0}\right)$ is not a transversal of $\mathcal{B}_{r_{0}}^{-}$. Therefore $\ell_{0}\left(r_{0}\right)$ is not a transversal, and thus not an extreme transversal, of the set $\left\{B\left(x_{i}, r_{0}-r_{i}\right), \ldots B\left(x_{n}, r_{0}-r_{n}\right)\right\}$ and would not have been computed by the nongeneric oracle at $r_{0}$. This contradicts the fact that $\ell_{0}\left(r_{0}\right) \in \mathcal{L}\left(r_{0}\right)$. Hence, the generic algorithm always performs a comparison whose corresponding polynomial vanishes at $r^{*}$.

To make this parametric searching efficient, we perform the generic simulation of the first phase of the oracle using a parallel implementation [15]. For each ball $B_{i}$, the first phase computes the region between the upper envelope and the lower envelope of two families of bivariate functions, using the algorithm of Agarwal et al. [3]. The only step in this algorithm that is nontrivial to parallelize is the construction of the overlay of two planar maps, each of which is a minimization diagram of a family of bivariate functions. The sequential algorithm in [3] uses a sweep-line algorithm to compute the overlay, which is difficult to parallelize. Instead, we compute the overlay using segment trees, which is easier to parallelize, as described in [4]. The parallel version of the overlay procedure runs in $O(\log n)$ time using $O\left(n^{2+\varepsilon}\right)$ processors, under Valiant's comparisons model, which is the model that is relevant for the generic simulation. Plugging this procedure into the algorithm of Agarwal et al. [3], we can compute the region lying between the upper and lower envelopes in $O\left(\log ^{2} n\right)$ time using $O\left(n^{2+\varepsilon}\right)$ processors, for any $\varepsilon>0$; see [3] for details. Executing this procedure for all balls of $\mathcal{B}$ in parallel, we can compute the set $\mathcal{L}$ of potential extreme transversals in $O\left(\log ^{2} n\right)$ time, using $O\left(n^{3+\varepsilon}\right)$ processors, for any $\varepsilon>0$.

Finally, simulating the parallel algorithm generically, as described in [15], we can compute a smallest enclosing cylinder of $\mathcal{B}$ in time $O\left(n^{3+\varepsilon}\right)$, for any $\varepsilon>0$. Hence, we obtain the following result:

Theorem 6.2. Given a set $\mathcal{B}$ of $n$ balls in $\mathbb{R}^{3}$, the minimum-radius cylinder containing (resp. intersecting) every ball of $\mathcal{B}$ can be computed in time $O\left(n^{3+\varepsilon}\right)$, for any $\varepsilon>0$. 


\section{An Approximation Algorithm}

Since computing a smallest enclosing cylinder exactly is expensive, it is desirable to design more efficient algorithms that solve the following approximation problem: Given a set $S$ of $n$ balls (or points) in $\mathbb{R}^{3}$ and a real parameter $\delta>0$, compute a cylinder containing (or intersecting) every ball (or point) in $S$, whose radius is at most $(1+\delta) r^{*}$, where $r^{*}$ is the radius of a smallest enclosing (or intersecting) cylinder of $S$. Schömer et al. [23] consider a somewhat different problem. They give an $O\left(\left(1+1 / \delta^{2}\right) n \log (1 / \delta)\right)$-time algorithm for computing a cylinder that contains $S$ and has radius at most $r^{*}+\delta \cdot \operatorname{diam}(S)$. If the shape of $S$ is sufficiently "round," then $r^{*}$ and $\operatorname{diam}(S)$ are comparable in size, so the output of the algorithm of [23] is comparable with what we want. However, if $S$ is "long and skinny" then $r$ * can be arbitrarily small compared with $\operatorname{diam}(S)$, in which case the value returned by the algorithm of [23] will be much larger than $(1+\delta) r^{*}$. We present an $O\left(\left(1+1 / \delta^{2}\right) n\right)$-time algorithm that computes a cylinder of radius at most $(1+\delta) r^{*}$ containing (or intersecting) $S$.

For the sake of simplicity, we describe the algorithm for the case when $S$ is a set of $n$ points in $\mathbb{R}^{3}$. The modifications for the more general case of balls are straightforward. Let $Q(s, r)$ be the axis-parallel cube of side $2 r$ centered at $s$, i.e.,

$$
Q(s, r)=\left\{p:\left|p_{x}-s_{x}\right|,\left|p_{y}-s_{y}\right|,\left|p_{z}-s_{z}\right| \leq r\right\} .
$$

For a direction $\mathbf{n}$, let $S^{(\mathbf{n})}$ denote the orthogonal projection of $S$ onto the plane $H_{\mathbf{n}}$ normal to $\mathbf{n}$ and passing through the origin. If a cylinder of radius $r$ with axis in direction $\mathbf{n}$ contains $S$, then there is a disk of radius $r$ within $H_{\mathbf{n}}$ that contains $S^{(\mathbf{n})}$. We now describe the algorithm.

\section{ALGORITHM: SMALL ENCLOSING CYLINDER}

1. Compute the diameter of $S$.

2. Perform a linear transformation on $S$ so that the two points $p=(0,0,0)$ and $q=(0,0,1)$ form a diametral pair, i.e., $p, q \in S$ and $\operatorname{diam}(S)=$ $d(p, q)$.

3. Compute the smallest value $r_{0}$ such that the set $\left\{Q\left(s, r_{0}\right) \mid s \in S\right\}$ of cubes admits a line transversal. Since a cube of side length $2 r$ has an inscribed ball of radius $r$ and a circumscribing ball of radius $\sqrt{3} r$, we have

$$
r^{*} / \sqrt{3} \leq r_{0} \leq r^{*}
$$

4. Set $v=\delta r_{0}$ and $b=\min \left\{4 \sqrt{3} r_{0}, \pi / 2\right\}$. Choose a set $\Delta$ of $O\left(1 / \delta^{2}\right)$ points on $K_{b}$, the spherical cap with angular opening $b$ and centered at the north pole $q$, so that, for any point $\mathbf{n} \in K_{b}$, there is a point $\mathbf{n}^{\prime} \in \Delta$ with $\angle \mathbf{n} p \mathbf{n}^{\prime} \leq v ; \Delta$ can be computed by drawing a grid of longitudes and latitudes on $\bar{K}_{b}$ of size $c / \delta \times c / \delta$, where $c>0$ is an appropriate absolute constant.

5. For each $\mathbf{n} \in \Delta$, compute the smallest disk, $D_{\mathbf{n}}$, enclosing $S^{(\mathbf{n})}$. Let $r_{\mathbf{n}}$ denote the radius of $D_{\mathbf{n}}$, and let $\ell_{\mathbf{n}}$ be the line passing through the center of $D_{\mathbf{n}}$ in direction $\mathbf{n}$.

6. Let $\mathbf{n}_{0} \in \Delta$ be a direction with the minimum value of $r_{\mathbf{n}_{0}}$. Then the cylinder of radius $r_{\mathbf{n}_{0}}$ with $\ell_{\mathbf{n}_{0}}$ as its axis is the desired cylinder. 
Lemma 7.1. The above algorithm computes a cylinder of radius $(1+\delta) r^{*}$ enclosing $S$.

Proof. Let $\mathbf{n}^{*}$ be the direction of the axis of a smallest cylinder enclosing $S$. We claim that $\mathbf{n}^{*} \in K_{b}$. Indeed, if $\mathbf{n}^{*} \notin K_{b}$, then the angle between the positive $z$-axis and $\mathbf{n}^{*}$ is more than $b$. Let $p^{*}, q^{*}$ be the orthogonal projections of $p$ and $q$ onto $H_{\mathbf{n}^{*}}$. Since $S$ is contained in a cylinder of radius $r^{*}$ whose axis is in direction $\mathbf{n}^{*}, d\left(p^{*}, q^{*}\right) \leq 2 r^{*}$. On the other hand,

$$
d\left(p^{*}, q^{*}\right)>\sin b \geq b / 2=2 \sqrt{3} r_{0} \geq 2 r^{*},
$$

a contradiction. Hence, $\mathbf{n}^{*} \in K_{b}$.

By construction, there is a direction $\mathbf{n}_{0} \in \Delta$ so that the angle between $\mathbf{n}^{*}$ and $\mathbf{n}_{0}$ is at most $v$. Let $w$ be a point of $S$, and let $w_{0}, w^{*}$ denote the corresponding projections of $w$ in $S^{\left(\mathbf{n}_{0}\right)}, S^{\left(\mathbf{n}^{*}\right)}$, respectively. By construction, we have $\left|w w_{0}\right|,\left|w w^{*}\right| \leq|w p| \leq 1$ (recall that the diameter of $S$ is assumed to be 1). Since the angle between the directed segments $w w_{0}$ and $w w^{*}$ is at most $v$, it follows that $\left|w_{0} w^{*}\right| \leq v$. Since $S^{\left(\mathbf{n}^{*}\right)}$ is contained in a disk of radius $r^{*}$, it follows that all the points of $S^{\left(\mathbf{n}_{0}\right)}$ are contained in a ball of radius $r^{*}+v \leq(1+\delta) r^{*}$; all these points being coplanar, they are also contained in a disk of radius $(1+\delta) r^{*}$. In other words, $r_{\mathbf{n}_{0}} \leq(1+\delta) r^{*}$. Let $c$ be the center of this disk. Then the cylinder of radius $r_{\mathbf{n}_{0}} \leq(1+\delta) r^{*}$ whose axis is the line passing through $c$ in direction $\mathbf{n}_{0}$ contains $S$.

The diameter of $S$ can be computed in $O(n \log n)$ randomized expected time [9], and $r_{0}$ can be computed in $O(n)$ time using the algorithm of Amenta [5]. For each $\mathbf{n} \in \Delta, D_{\mathbf{n}}$ can be computed either in linear worst-case time using Megiddo's deterministic algorithm [16], [17] or in linear expected time using the randomized algorithm of Matoušek et al. [14]. Hence, the overall expected running time of this randomized implementation of the algorithm is $O\left(n \log n+n / \delta^{2}\right)$. A closer look at the proof of Lemma 7.1 shows that in Step 1 it suffices to compute a pair of points $p, q \in S$ that are separated by a distance of at least $\operatorname{diam}(S) / 2$. Such a pair of points can be easily computed deterministically in worstcase time $O(n)$ : choose an arbitrary point $p$ of $S$ and compute its farthest neighbor $q$ in $S$ (if there is more than one such point, choose any of them); by the triangle inequality, $d(p, q) \geq \operatorname{diam}(S) / 2$. This makes the algorithm fully deterministic, and improves its total running time to $O\left(\left(1+1 / \delta^{2}\right) n\right)$. Moreover, the algorithm can be easily extended to the case in which $S$ is a set of balls instead of a set of points. One notable difference is that Step 5 has to be replaced by the algorithm of Megiddo [18] for finding, in linear time, the smallest disk containing a given set of disks. Hence, we obtain the following result:

Theorem 7.2. Given a set $S$ of $n$ balls in $\mathbb{R}^{3}$ and a parameter $\delta>0$, a cylinder containing (or intersecting) every member of $S$, whose radius is at most $1+\delta$ times that of a smallest enclosing (or intersecting) cylinder of $S$, can be computed in (deterministic) time $O\left(\left(1+1 / \delta^{2}\right) n\right)$. 


\section{Conclusion}

In this paper we obtained near-cubic bounds for the complexity of the space of line transversals of a collection $\mathcal{B}$ of $n$ balls in $\mathbb{R}^{3}$ or of $n$ homothets of any convex body of constant description complexity. We also developed near-cubic algorithms for determining whether $\mathcal{T}(\mathcal{B})=\emptyset$ and for computing a smallest cylinder enclosing (or intersecting) every member of $\mathcal{B}$. We finally presented a linear-time approximation algorithm for computing a small cylinder enclosing $\mathcal{B}$. The main open problem is to obtain near-cubic bounds for the case of collections of general convex sets of simple shape in $\mathbb{R}^{3}$. We conjecture that such bounds hold in fairly general situations, but the proof techniques used in this paper do not seem to extend to more general sets. In the light of the discussion in Section 2, this conjecture would automatically be established if one manages to extend the results of [3] concerning overlay of envelopes to four dimensions.

Another open problem is to extend the results of this paper to higher dimensions. Since lines in $d$ dimensions have $2 d-2$ degrees of freedom, we conjecture that the complexity of the space of line transversals of a collection of $n$ balls, or of convex polyhedra with a total of $n$ faces (of all dimensions), in $\mathbb{R}^{d}$ is close to $O\left(n^{2 d-3}\right)$.

\section{References}

1. P.K. Agarwal, On stabbing lines for convex polyhedra in three dimensions, Comput. Geom. Theory Appl. 4 (1994), 177-189.

2. P.K. Agarwal, B. Aronov, and M. Sharir, Computing lower envelopes in four dimensions with applications, SIAM J. Comput. 26 (1997), 1714-1732.

3. P.K. Agarwal, O. Schwarzkopf, and M. Sharir, The overlay of lower envelopes in 3-space and its applications, Discrete Comput. Geom. 15 (1996), 1-13.

4. P.K. Agarwal, M. Sharir, and S. Toledo, New applications of parametric searching in computational geometry, J. Algorithms 17 (1994), 292-318.

5. N. Amenta, Helly-type theorems and generalized linear programming, Discrete Comput. Geom. 12 (1994), 241-261.

6. D. Avis, J.-M. Robert, and R. Wenger, Lower bounds for line stabbing, Inform. Process. Lett. 33 (1989), 59-62.

7. D. Avis and R. Wenger, Polyhedral line transversals in space, Discrete Comput. Geom. 3 (1988), 257-265.

8. U. Babu, J. Raja, and R. Hocken, Sampling methods and substitute geometry algorithms for measuring cylinders in coordinate measuring machines, Proc. Annual Meeting of the ASPE, 1993.

9. K. L. Clarkson and P. W. Shor, Applications of random sampling in computational geometry, II, Discrete Comput. Geom. 4 (1989), 387-421.

10. A. Forbes, Generalized regression problems in metrology, Numer. Algorithms 5 (1993), 523-533.

11. J.E. Goodman, R. Pollack, and R. Wenger, Geometric transversal theory, in: New Trends in Discrete and Computational Geometry (J. Pach, ed.), Springer-Verlag, Heidelberg, 1993, pp. 163-198.

12. J.E. Goodman, R. Pollack, and R. Wenger, Bounding the number of geometric permutations induced by $k$-transversals, Proc. 10th ACM Symp. on Computational Geometry, 1994, pp. 192-197.

13. K. Kedem, R. Livne, J. Pach, and M. Sharir, On the union of Jordan regions and collision-free translational motion amidst polygonal obstacles, Discrete Comput. Geom., 1 (1986), 59-71.

14. J. Matoušek, M. Sharir, and E. Welzl, A subexponential bound for linear programming, Algorithmica 16 (1996), 498-516.

15. N. Megiddo, Applying parallel computation algorithms in the design of serial algorithms, J. Assoc. Comput. Mach. 30 (1983), 852-865.

16. N. Megiddo, Linear-time algorithms for linear programming in $R^{3}$ and related problems, SIAM J. Comput. 12 (1983), 759-776. 
17. N. Megiddo, The weighted Euclidean 1-center problem, Math. Oper. Res. 8 (1983), 498-504.

18. N. Megiddo, On the ball spanned by balls, Discrete Comput. Geom. 4 (1989), 605-610.

19. M. Pellegrini, Stabbing and ray shooting in 3-dimensional space, Proc. 6th Ann. ACM Symp. on Computational Geometry, 1990, pp. 177-186.

20. M. Pellegrini, Ray shooting on triangles in 3-space, Algorithmica 9 (1993), 471-494.

21. M. Pellegrini, Lower bounds on stabbing lines in 3-space, Comput. Geom. Theory Appl. 3 (1993), 53-58.

22. M. Pellegrini and P. Shor, Finding stabbing lines in 3-space, Discrete Comput. Geom. 8 (1992), 191-208.

23. E. Schömer, J. Sellen, M. Teichmann, and C.K. Yap, Efficient algorithms for the smallest enclosing cylinder problem, Proc. 8th Canadian Conf. on Computational Geometry, 1996, pp. 264-269.

24. M. Sharir, Almost tight upper bounds for lower envelopes in higher dimensions, Discrete Comput. Geom. 12 (1994), 327-345.

25. M. Sharir and P.K. Agarwal, Davenport-Schinzel Sequences and Their Geometric Applications, Cambridge University Press, New York, 1995.

26. V. Srinivasan, How tall is the pyramid of Cheops?...And other problems in computational metrology, SIAM News 29(3) (1996), 8-9.

27. K. Suresh and H. Völcker, New challenges in dimensional metrology: a case study based on "size," Manufacturing Rev. 7 (1994), 304-311.

28. R. Wenger, Upper bounds on geometric permutations for convex sets, Discrete Comput. Geom. 5 (1990), 27-33.

29. R. Wenger, Helly-type problems and geometric transversals, in CRC Handbook of Discrete and Computational Geometry (J. Goodman and J. O’Rourke, eds.), CRC Press, Boca Raton, FL, 1997, pp. 63-82.

Received May 23, 1997, and in revised form October 20, 1997. 